\title{
Improved Design of an Adaptive Massive MIMO Spherical Antenna Array
}

\author{
Mouloud Kamali ${ }^{1}$ \\ Electrical engineering department, National Engineering \\ School of Carthage, Tunis Carthage-Tunisia \\ Analysis and Processing of Electrical and Energic Systems
}

\author{
Adnen Cherif $^{2}$ \\ Analysis and Processing of Electric and Energetic Systems \\ Faculty of Sciences of Tunis \\ ELMANAR II, PB.1060, Tunisia
}

\begin{abstract}
Massive capacity and connectivity are the main boundaries towards standing the Internet of Everything (IoE) basis and defining modern wireless generation requirements. These needs cannot be achieved by already deployed phased array antenna in terms of distributed and oriented geometry, dimensions and design. We propose in the present paper an innovating massive multiple input multiple output (MIMO) spherical array network aiming to draw a new three-dimensional configuration to enhance the beam steering, improve bandwidth, total capacity and the scan flexibility. Resolved issues in concordance with $5 \mathrm{G}$ requirements are adaptive massive MIMO by using millimeter-wave antenna arrays, small cell design and definition of recommended operational frequency considering the International Telecommunication Union (ITU) norms and directives. The new geometric forms of spherical smart antenna could easily scan all 3D space, ensure higher capacity and reach tens of Giga bit per second (Gbps) value besides eradicating energy wastage aspect of Beam Division Multiple Access (BDMA) in base stations. Mathematical design is detailed and performed simulation results are presented using MATLAB software Tool.
\end{abstract}

Keywords-Adaptive spherical antenna; beam division multiple access BDMA; massive multiple input multiple output MIMO; millimeter-wave mm-wave

\section{INTRODUCTION}

The transported information via mobile networks, counting data, voice and internet are continuously growing with a drastic quality and quantity. The main polemic topic is that older generations of wireless communication from $0 \mathrm{G}$ to $4 \mathrm{G}$ doesn't support the new information flux.

Unceasingly growing bit rate bulk either at transmission or at reception's end, Besides the substantial strengthening of interrelated networks fitting the base of a new architype developing from the significant objects link such as robots, machines and vehicles. This paradigm is well known in IoE included in $5 \mathrm{G}$.

The most attractive key ascertainments resolved by $5 \mathrm{G}$ are: high mobility, slight latency besides the massive connectivity and capacity.

The next expected imminent event of mobile communications is going to be unrelated to nowadays usage. Even though, Request for mobile broadband will remain to increase, fundamentally determined by the new usage of ultrahigh definition video and $\mathrm{K}$ screen modes, we have already seen the expanding influence of cars, robots and devices that must able to attain and outline themselves to achieve the human necessities. There will be surely many not yet perceived, several performs appear in horizon and engender new materials that communications networks must pact with the demanded cost efficiency.

Essentially, there is tendencies concentrating on how to support the vicissitudes of real-world mobility, virtual mobility, 4th industrial revolution, Solid-performance mobile infrastructure and numerous other related domains such as healthcare, Rail network and automotive, Manufacturing and logistics... . Consequently, the overall annexed industry will be affected by this ongoing migration to the $5 \mathrm{G}$ telecom mobile.

As a result, A comprehensive mobile data traffic is increasingly growing in term of new connected everything number [1], including pedestrians, vehicles, healthcare [2] and machines. To upgrade massive connectivity capacity and insignificant latency we should guarantee; 1000X higher mobile data volume per area 10-100X higher [3], Number of connected devices for Internet of Things IoT [4], 10-100X higher typical user data rate [5], 10X longer battery life for low power Machine to Machine M2M Communications, 5X reduced End to End E2E [6] latency.

To accommodate this new trend, the wireless network infrastructure as we said needs considerable upgrades beyond the already installed capabilities. Afterward, many researchers are focusing on several aspects like operable carrier frequencies and service guidelines. The gigantic accessible bandwidth for highly multidirectional wireless links must be in accordance with future generations of wireless mobile networks.

A special interest is imposed to be considered for the channel characteristics, which, in many situations is not well recognized because of the needed bandwidth range. Obviously, at these higher frequencies, $\mathrm{GHz}$ signals are working as well as the distance of serving others are nearby some hundred meters.

In literature, researchers are mainly focusing on the modeling and design aspects of millimeter-wave, channel model, energy efficiency, new antenna geometries, MIMO models, Mathematical architecture modeling, Multi-Beam antenna and coders. Hereunder we cite some of them were:

Authors in [7] focus on the impact of blockage of mmWave signals in spatial reduced coverage owing penetration through the human hand, body and vehicle at $28 \mathrm{GHz}$. They 
use statistical blockage models aiming to reproduce last cited impacts (hand, human body and vehicle). Their results show that the time-scales corresponding to blockage on the order of some hundreds of milliseconds. Also, a robust of mm-Wave beamforming to handle blockage studies have been presented.

In [8] authors propose a 3D spatial channel model controlling, aiming to evaluate practically conceivable boundaries of massive MIMO base stations standardized by the third Generation of mobile networks 3G. They consider the Base Stations BSs completely loaded and different configurations of active User Ends UEs per cell besides, they present the established statistical approach to reduce to half the compliance distance in comparison to the traditional technique.

While in [9] authors investigate the problem of designing a control channel in a $5 \mathrm{G}$ system. Their control chain includes the transmission, under the severe latency and reliability of a short data packet comprising a trivial information payload, over a propagation channel that offers limited frequency diversity and no time diversity. They present an achievability bound, built upon the random-coding union bound which trusts on quadrature phase-shift FSK keying modulation and pilotassisted transmission to estimate the diminishing channel, and scaled nearest-neighbor decoding at the receiver. They determine the number of pilot symbols that should be transmitted to optimally occupy between channel-estimation errors and rate loss related to pilot overhead. Besides, they underline the importance of using multiple antennas at the transmitter and/or the receiver.

In the same axis [10] presents energy-efficient resource allocation in multiple antenna wiretap channels is investigated, focusing on maximum power and minimum privacy capacity/rate constraints. Two energy-efficiency metrics were optimized, knowing the secrecy energy efficiency and the secret-key energy efficiency. If the valid receiver and the listener have a single antenna, and the transmitter has multiple antennas, the global solution provided by the authors is expressed by a simple formula resolved by iterative algorithms which can determine the global maximum of the secret-key energy efficiency and candidate solutions related to secrecy energy efficiency maximization problem.

Though in [11] they present a novel geometry-based statistical model for small-scale non-wide-sense stationary uncorrelated scattering mobile-to-mobile Rayleigh fading channels. Their model is based on the plane wave propagation to capture the temporal evolution of the propagation delay and Doppler shift of the received multipath signal which is different from spherical wave propagation statistical model method, which yield more realistic case. They consider an arbitrary geometric configuration of the propagation zone. They derive general expressions for the most important statistical quantities of non-stationary channels including the frequency correlation function, the local scattering function, the frequency dependent time and the Doppler profiles.

In [12], the author presents 3D vehicle MIMO antenna array model for vehicle-to-vehicle (V2V) communication environments. A spherical wave front is used in the proposed model as an alternative of the plane wave front supposition used in the conventional MIMO channel model. Using the proposed V2V channel model, they first derive the closed-form expressions for the joint and marginal probability density functions of the angle of departure at the transmitter and angle of arrival at the receiver in the azimuth and elevation planes. They analyse in addition the time and frequency crosscorrelation functions for different propagation paths. An expression of Doppler spectrum related to the relative motion between the mobile transmitter and mobile receiver has been derived from the proposed model.

Unlike [13] they reflect the possibility of locating a system where antenna arrays are organized as a Large, Intelligent Surface (LIS) electronically active with integrated electronics and wireless communication making the entire situation "intelligent". They extensively discuss the impact of centralized and distributed deployments of LIS and show that a distributed deployment of LIS could enlarge the coverage and improve the global positioning performance.

In [14], A spatially correlated large antenna array operating at $\mathrm{mm}$-Wave frequencies are considered. The properties of the Power Elevation Spectra (PES) on the meeting massive MIMO properties are then demonstrated by defining and deriving a diagonal dominance metric. Statistically, the properties of the antenna element Mutual Coupling (MC) are exposed on the active spatial correlation (SC), mm-Wave user rate, and eigenvalue structure for different antenna topologies. It is determined that even if MC could meaningfully decrease SC for side-by-side dipole antenna elements, the modification in antenna real gain and consequently, signal-to-noise ratio (SNR) caused by $\mathrm{MC}$ becomes a governing effect and eventually limits the antenna array performance. The mm-Wave user rating system with hybrid beamforming, using an orthogonal matching pursuit algorithm, is then shown for different antenna topologies with dipole and cross polarized (x-pol) antenna elements.

While [15] they establish a full 3D channel model to support the performance analysis and evaluation of active antenna array based on wireless communication systems. they analyse and compare the impact of three different down-tilt methods employed in active antenna array antennas, electrical down-tilt, mechanical down-tilt, hybrid down-tilt, on the antenna patterns, which would notably impact the performance of mobile wireless communication systems. A comparison of the wireless communication system throughput of $2 \mathrm{D}$ and $3 \mathrm{D}$ wireless channel models has been developed. The system performance in terms of capacity and coverage with different active antenna arrays under the $3 \mathrm{D}$ channel has been investigated. Significant gains in coverage and capacity for individual antennas with a narrow beam of the vertical patterns have been observed using down-tilt optimization. But it may not lead to a distinguished gain for individual antennas with relatively large beam-width of the vertical patterns.

In the case of [16] a mathematical model is provided of a novel hybrid precoding architecture, and an efficient alternating descent algorithm is developed to jointly design the analog and digital precoders for the last proposed hybrid precoding scheme. Numerical results obtained demonstrate that their proposed precoding scheme with multi-feed reflect array 
antennas achieves much better precoding performance than its sub-connected counterpart with phased array antennas.

While, in [17] a massive MIMO is presented as an extension of traditional MIMO technology, it subsequently improves the throughput rate, energy efficiency, the link reliability and data transmission rate. It also improves through a lot of increasing the antenna communication number, by means of very duplex communication mode, which correspond to a high spectrum efficiency system.

In [18] the achievable sum-rate of the proposed beamdomain full-duplex (FD) massive MIMO transmission scheme is analysed and the joint user and BS power allocation scheme are proposed to optimize the system achievable sum-rate. Their simulation results show that the proposed beam-domain FD transmission scheme outperforms existing time division duplex and frequency division duplex massive MIMO and FD massive MIMO transmission schemes on the spectral efficiency performance.

Whereas [19] presents interference moderation for multibeam antenna subsection modulation via side lobe level reduction is introduced. A method for designing thinned arrays with minimum side lobe levels for antenna subset modulation is introduced and generalized for multi-beam antenna subset modulation. A new variable constraint is applied to the optimization problem to control the localization of optimum solution within the antenna array. Two solutions are introduced, convex optimization combined with local search and local search assisted genetic algorithm.

Although [20] is a formalization of hybrid precoders design as a block-sparse reconstruction problem and a minor complexity algorithm for finding precoders is proposed based on the greedy sequence clustering. To assure the performance of this algorithm, an estimation method for the number of the blocks and radio frequency chains is proposed. Under this outline, for the analog precoder the number of the phases needed to be quantized is only two times minus one of that of all blocks, which remains unchanged and even the number of chains increases. They demonstrate that their proposed algorithm could achieve the near-optimal performance and save the feedback overhead by utilizing the block sparsity information.

All these cited works have some drawbacks existing in:

- Studies don't match mm-Waves according to the atmospheric and molecular absorption at mm-Wave frequencies but more exactly $\mathrm{cm}$-waves.

- 4G multi-cell and multi-layer optimisation doesn't match the case of $5 \mathrm{G}$ there is a new central universal resource management.

- The capacity offered by PCM modulation is insignificant regarding QAM, which ought to be not less than 256 bits constellation.

- The energy efficiency increases when the distance between BS and UE is important. Thus, the capacity diminishes. Nevertheless, in $5 \mathrm{G}$ there are small cells when the capacity is higher and consumed energy is slighter.

Our novelty presented in this paper compared to already cited references was resumed in three main originalities:

First, a new spherical topology in which the number of elements is significantly boosted, embedded in a massive MIMO. Second, the frequency is elevated. Third, cell dimensions become smaller. All this, knowing that in urban zones, already installed antenna array geometries are limited to direct visibility and don't deal in the case of high buildings connection requests. Spherical antenna array with Massive MIMO ensures as a result in dense and vertical buildings agglomerations the coverage quality with the same probability for $3 \mathrm{D}$ space.

This paper is divided into 3 sections. The first section is the introduction, the second section treats the mathematical modelling of the spherical antenna, the third purposes to present performed simulation results and its related discussion and finally the conclusion and the main perspectives have been done.

\section{Mathematical Modeling of The Spherical ANTENNA}

$E_{\text {total }}=E_{\text {single element at reference point }} * \mathrm{AF}$

The total electrical field is the multiplication of the single element reference point field by the array factor.

$$
F_{\text {total }}(\theta, \phi)=F_{\text {element }}(\theta, \phi) * A F_{\text {array }}(\theta, \phi)
$$

$\theta$ is the elevation angle and $\varphi$ is the azimuth angle.

\section{A. Single Element at Reference Point of the Electrical Field}

Represented by Fig. 1 hereunder the Microstrip element model dedicated for spherical Antenna.

The free-space wavenumber $K=\frac{2 \pi}{\lambda}$

The patch Microstrip: length $L$, width $W$,

where $W=L=\frac{\lambda}{2}$

The magnitude of the fields, given by:

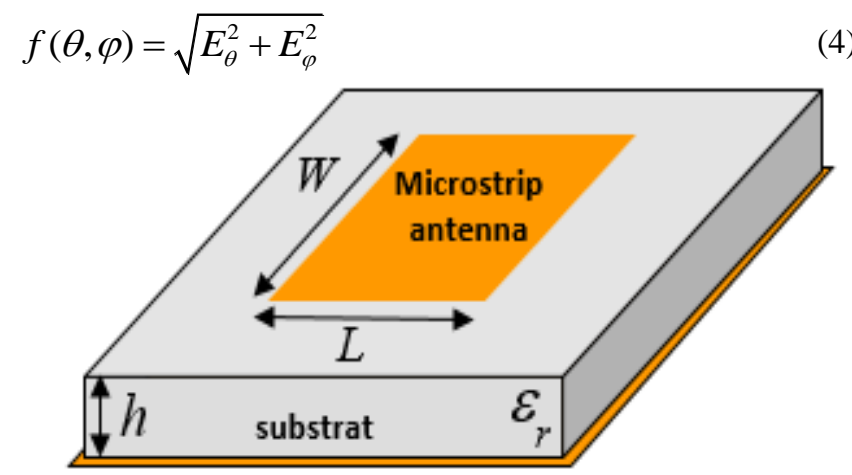

Fig. 1. Microstrip element model dedicated for spherical Antenna 


$$
\begin{aligned}
E_{\theta} & =\frac{\sin \left(\frac{K W \sin \theta \cos \varphi}{2}\right)}{\frac{K W \sin \theta \cos \varphi}{2}} \cos \left(\frac{K L \sin \theta \cos \varphi}{2}\right) \cos \varphi \\
E_{\varphi} & =\frac{\sin \left(\frac{K W \sin \theta \cos \varphi}{2}\right)}{\frac{K W \sin \theta \cos \varphi}{2}} \cos \left(\frac{K L \sin \theta \cos \varphi}{2}\right) \cos \theta \sin \varphi
\end{aligned}
$$

\section{B. Array Factor}

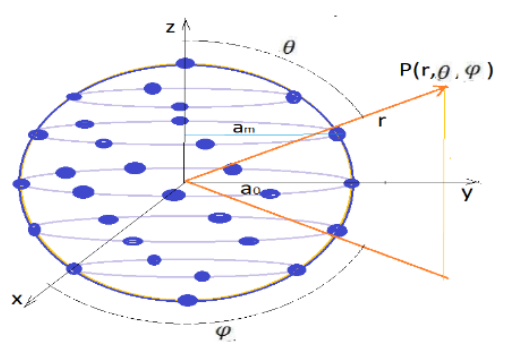

Fig. 2. Antenna topology and disposition for spherical antenna array

Conformal arrays are specifically conceived in a manner to imitate a surface. The radiating elements of the spherical array as shown in Fig. 2 could be easily embedded in the tight curved surface, thus automatically reduce disagreements by the installed components. The spherical shaped antenna array could be preserved as one of the greatest elements of the conformal array. The most striking feature of spherical array is as follows:

- All points in the far-field will perceive the same environment with a spherical array because of its symmetrical elements disposition due to the radiation pattern that will persist like the considered far-field point is enthused over the space.

- These arrays could be handled to reach multiple-beams and adaptive patterns redesigning based on its electronic beam routing and signal processing capabilities.

$$
\begin{aligned}
& A F_{\text {Spherical }}(\theta, \varphi)= \\
& \sum_{n=-N}^{N} \sum_{m=1}^{M}\left[I_{m n} e^{\left(j \mathrm{Ka}_{n} \sin \theta \cos \left(\varphi-\varphi_{m n}\right)+j \varphi_{m}\right)+\mathrm{jKd}_{n} \cos \left(\theta+\beta_{n}\right)}\right]^{(7)} \\
& +e^{\mathrm{jka} \cos \theta}+e^{-\mathrm{jka} \cos \theta} \\
& \mathrm{a}_{\mathrm{n}}=\sqrt{\mathrm{a}_{0}^{2}-d_{n}^{2}}
\end{aligned}
$$

$I_{m n}$ is the current excitation for $\mathrm{m}^{\text {th }}$ antenna element of $\mathrm{n}^{\text {th }}$ circular array.

$\mathrm{K}$ is the propagation constant,

$\theta$ is the elevation angle,

$\phi$ is the azimuth angle,

$\phi_{m n}$ is the azimuth position of $\mathrm{m}^{\text {th }}$ antenna element on $\mathrm{n}^{\text {th }}$ circular array.

$a_{n}$ is the radius for $n^{\text {th }}$ circle of spherical array.

$\mathrm{a}_{0}$ is the radius of spherical array. $\phi_{m}$ is the beam steering phase angle in azimuth direction. $d_{n}$ is the distance of $\mathrm{n}^{\text {th }}$ circular array from reference circular array at the origin.

$\beta_{n}$ is the progressive phase shift between nth and reference circular array.

To design the spherical geometry, as circular arrays must be settled in a linear manner. Fig. 3 describes the disposition of the antenna array.

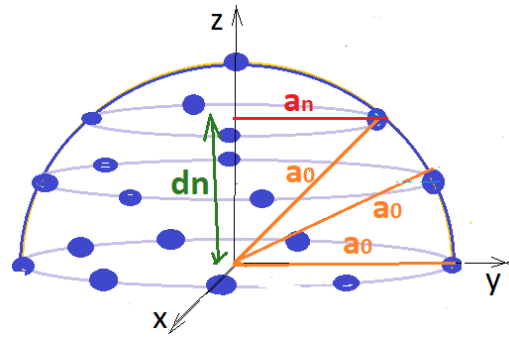

Fig. 3. Antenna topology and disposition

\section{Total Electrical Field Magnitude}

$$
\begin{aligned}
& E_{\text {total }}=\sqrt{E_{\theta}^{2}+E_{\varphi}^{2}} * \\
& {\left[\sum_{n=-N}^{N} \sum_{m=1}^{M}\left[I_{m n} e^{\left(j \mathrm{Ka}_{n} \sin \theta \cos \left(\varphi-\varphi_{m n}\right)+j \varphi_{m}\right)+\mathrm{jd}_{n} \cos \left(\theta+\beta_{n}\right)}\right]\right.} \\
& \left.+e^{\mathrm{jka}_{0} \cos \theta}+e^{-\mathrm{jka}_{0} \cos \theta}\right]
\end{aligned}
$$

\section{SimUlation RESUltS AND DisCUSSION OF MASSIVE MIMO AND MMWAVE SPHERICAL ANTENNA ARRAYS}

Filling a spherical array arranged one above the other, with the same separation distance between them, such as the cylindrical array wherein the antenna elements form a circular surface area equivalent to a disk array of antenna settled in multiple layers as shown in Fig. 3 and Fig. 4.

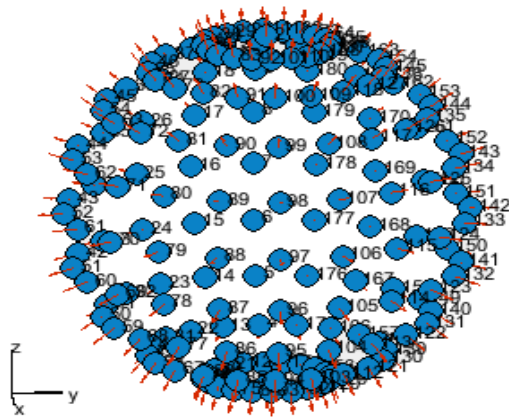

Fig. 4. Antenna topology and disposition of spherical Antenna

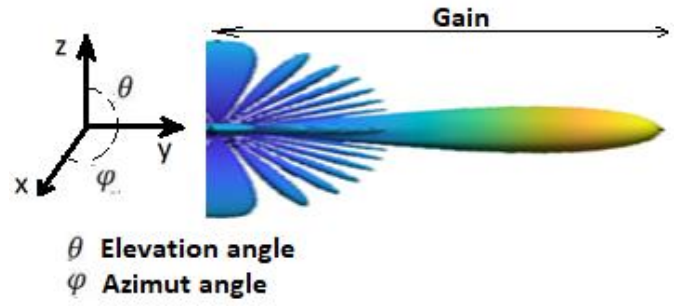

Fig. 5. Narrow beam width power for spherical Antenna topology 
In Fig. 5 the beam direction is commanded by azimuth and elevation angle. The power is defined by the distance between the user and the antenna demonstrated by the beam width.

In Fig. 6 a three-dimensional sight of array directivity at $90^{\circ}$ azimuth angle and $0^{\circ}$ elevation working at $73 \mathrm{Ghz}$ frequency indicating that the beams divide between requesting users. The yellow color matches $20 \mathrm{dBi}$ directivity where stated support users.

3D Directivity Pattern $73 \mathrm{GHz}$ steered at $90 \mathrm{Az}, 0 \mathrm{EI}$

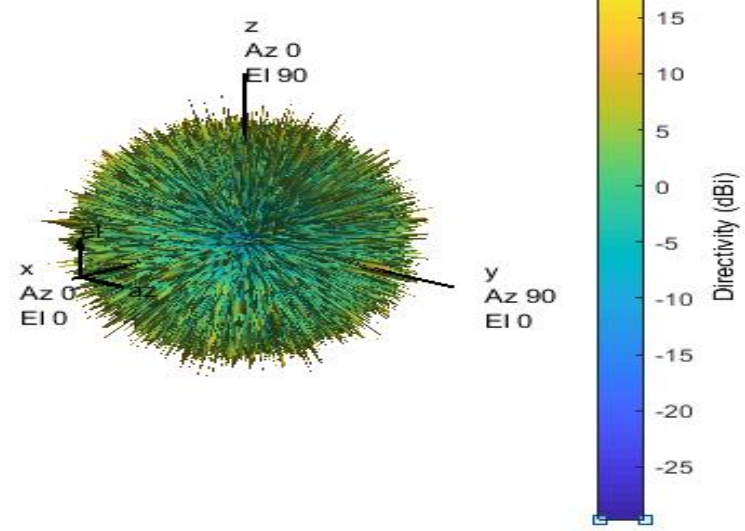

Fig. 6. Pattern power in 3D of the spherical Anenna

TABLE I. ARRAY CHARACTERISTICS

\begin{tabular}{|l|l|}
\hline Array directivity & $22.42 \mathrm{dBi}$ at $0^{\circ} \mathrm{Az}, 0^{\circ} \mathrm{El}$ \\
\hline Spherical Array Span & Rsphere $=4.5 \mathrm{~cm}$ \\
\hline Number of elements & Nantenna $=182($ massive MIMO) \\
\hline
\end{tabular}

Table 1 illustrates the array directivity, array span and the number of deployed antennas on our stated topology.

Fig. 7 labelled a cut section of the overall directivity pattern at the same azimuth associated to $0^{\circ}$ elevation angle.

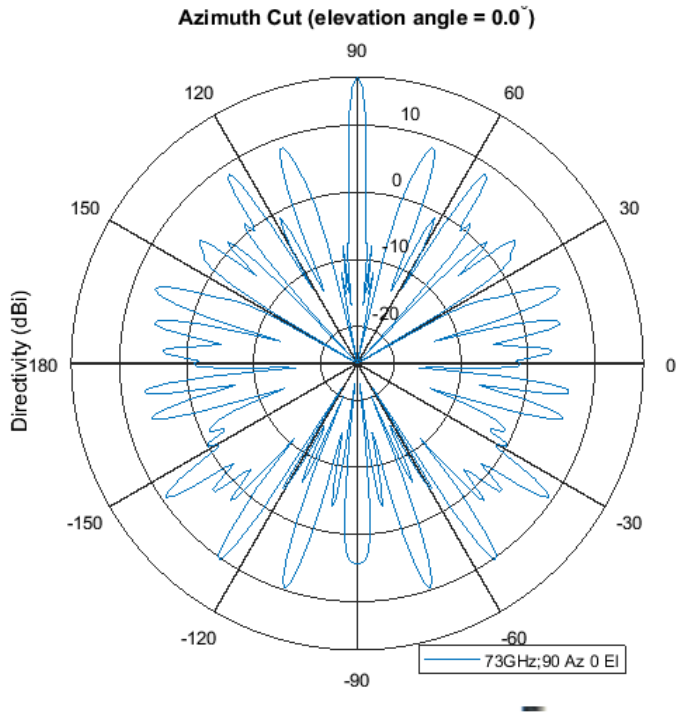

Fig. 7. Azimuth cut elevation angle of spherical Anenna

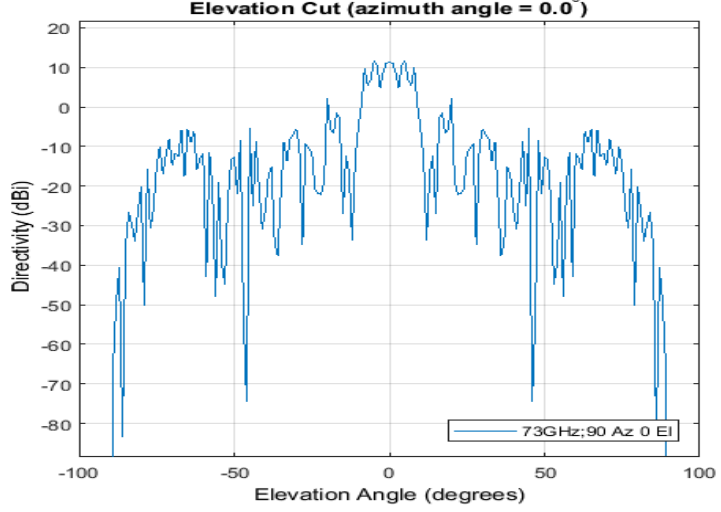

Fig. 8. Elevation cut angle of spherical Anenna

Fig. 8 shows the spherical system antenna Elevation cut angle as given in the design; the excitation coefficients, inter element arrangement, magnitude and phase, and angular partings are reformed in a way to acquire the required pattern in term of directivity strengthening, side lobe reduction, pattern nulling and shaping.

Fig. 9 describes a cut section of the overall directivity at elevation cut related to azimuth angle $0^{\circ}$. The main supported user is at $90^{\circ}$ azimuth as we visualize the maximum received power is confined to the cibled point in concordance with spherical coordinates all around the space.

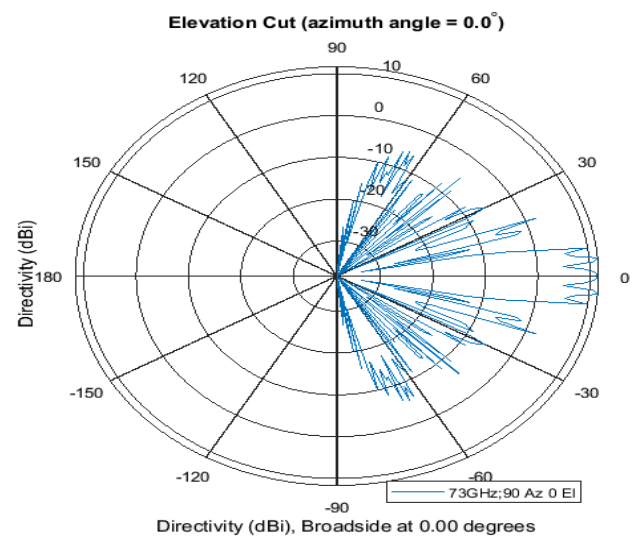

Fig. 9. Elevation cut Azimuth angle of spherical Anenna

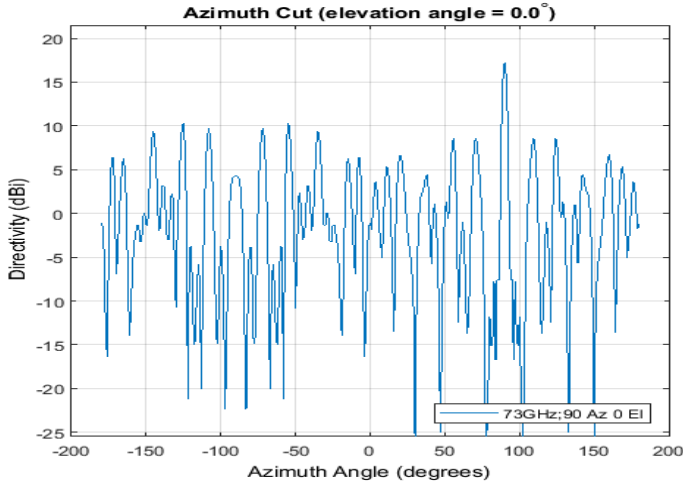

Fig. 10. Azimuth cut angle of spherical Anenna 
The last figure, Fig.10 presents a cut section of the overall directivity at $90^{\circ}$ azimuth cut associated to elevation angle $0^{\circ}$. The served user is at $90^{\circ}$ azimuth as we visualize the maximum received power is axed to the cibled point in concordance with spherical coordinates all over the space.

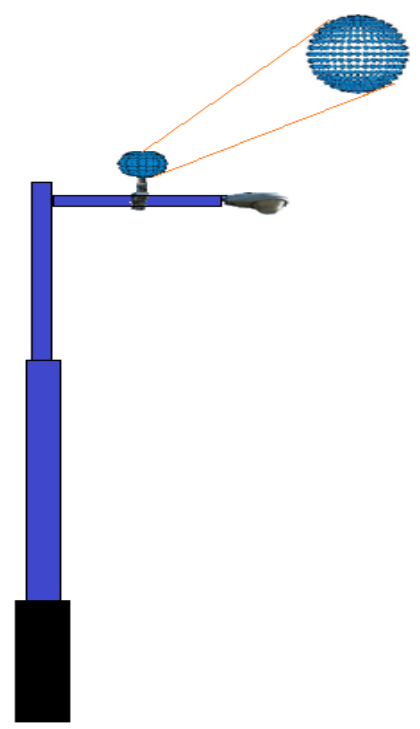

Fig. 11. Example of small cells on Lamposts of spherical Antenna

Figure 11 demonstrates a prototype disposition tied to lampposts of the spherical antenna array.

TABLE II. SUMMARY OF MATLAB VALUES

\begin{tabular}{|c|c|c|c|c|c|c|}
\hline \multicolumn{2}{|c|}{$F=73 \mathbf{G H z}$} & \multicolumn{5}{|c|}{182 antenna (massive MIMO) } \\
\hline $\begin{array}{c}\varphi_{(\text {azimut }):} \\
\left(-180^{\circ} \text { to }+180^{\circ}\right)\end{array}$ & $\mathbf{0}$ & 30 & 60 & 90 & 120 & 180 \\
\hline $\begin{array}{l}\theta_{(\text {(elevation) }} \\
\left(-90^{\circ} \text { to } 90^{\circ}\right)\end{array}$ & $\mathbf{0}$ & 15 & 30 & 45 & 60 & 90 \\
\hline $\operatorname{Pr}[\mathrm{dBi}]$ & 22.83 & 22.32 & 22.46 & 22.59 & 22.61 & 22.51 \\
\hline
\end{tabular}

- Link $73 \mathrm{GHz}$ Spherical properties:

- $\mathrm{F}=73 \mathrm{GHz}$.

- $\lambda=4 \times 10^{-3} \mathrm{~m}=4 \mathrm{~mm}$.

- $\mathrm{d}=\lambda / 2=2 \times 10^{-3} \mathrm{~m}=2 \mathrm{~mm}$.

- $\mathrm{R}_{\text {sphere }}=45 \times 10^{-3} \mathrm{~m}=4.5 \mathrm{~cm}$.

Table 2 represents the numerical simulation values in MATLAB. A contingent of the user locates the 3D spherical antenna array in content of users demand with maximal power of $22 \mathrm{dBi}$ thanks to the substantial number of adaptive antenna elements. Hence, beams are most directives and narrow $\mathrm{P}(\mathrm{r}$, $\theta, \varphi)$

An antenna matrix in the base station BS $(2 \times 2,3 \times 3,4 \times 4 \ldots)$ [21] edges the power depending only on the demanding user.

Hence the power is adjusted since exclusively demanding users are attended. Then, Services price will be more redressed for both sides, the operators and customers besides the omission of electromagnetic noise.

\section{CONCLUSION}

Along this paper, we have modelled mathematically a spherical antenna array with Massive MIMO, which could offer several benefits for migration into $5 \mathrm{G}$ mobile in urban zones, when cylindrical array antenna is considered to be functionally limited to direct visibility and not for high buildings cases. Spherical antenna array with Massive MIMO ensures as a result in dense and high agglomerations with the same probability for 3D space:

a) Largely increase the link reliability and the data rate.

b) Massive MIMO ensures:

- a deterministic channel matrix.

- could be constructed with cheap, low-power units.

- simplify the multiple access layer.

- contain systems with hundreds, even thousands of antennas could enable several-gigabit rate transmissions at high spectral efficiency.

However, numerous technical tasks have to be undertaken in realizing such large MIMO systems. The solution which enables 5G migration is firstly increasing the number of antenna elements using a spherical topology that means a Massive-MIMO system. Use a millimeter wavelength and small cells implicitly a low power consumption due to the adaptive array antenna.

Inspite of the spherical geometry effictiveness there is a little limitation for connectivity in very high speed case. Indeed, Connectivity at $1200 \mathrm{Km} / \mathrm{h}$ speed, users inside an airplane probably lose the network connection due to the absence of a a complementary assist as a Low Earth Orbit (LEO) satellite base station. Thus, a satellite support must be taken into account as a future work.

\section{REFERENCES}

[1] Marwan A. Al-Namari, Ali Mohammed Mansoor, "A Brief Survey on 5G Wireless Mobile Network", International Journal of Advanced Computer Science and Applications, Vol. 8, No. 11, 2017.

[2] Farah Nasri, Abdellatif Mtibaa, "Smart Mobile Healthcare System based on WBSN and 5G", International Journal of Advanced Computer Science and Applications, Vol. 8, No. 10, 2017.

[3] Nisha Panwar, Shantanu Sharma, and Awadhesh Kumar Singh, "A Survey on 5G: The Next Generation of Mobile Communication*", Elsevier Physical Communication, Volume 18, Part 2, pp. 64-84, March 2016.

[4] Mohammad Saeid Mahdavinejad , Mohammad reza Rezvana , Mohammad amin Barekatain, Peyman Adibi, PayamBarnaghi, Amit P.Sheth, "Machine learning for Internet of Things data analysis: A survey“, Digital Communications and Networks, In Press, 12 October 2017.

[5] Chun-Nan Liu, "Trend, technology and architecture of small cell in 5G era“, IEEE, VLSI Technology, Systems and Application (VLSI-TSA), 2016 International Symposium on, 25-27 April 2016 Hsinchu, Taiwan, pp. 1-2, May 2016.

[6] Ekram Hossain; Monowar Hasan, "5G cellular: key enabling technologies and research challenges ",IEEE Instrumentation \& Measurement Magazine, Volume 18, Issue 3, pp. 11 - 21, June 2015.

[7] Vasanthan Raghavan, Lida Akhoondzadeh-asl, Vladimir Podshivalov, Joakim Hulten, M. Ali Tassoudji, Ozge Hizir Koymen, Ashwin Sampath, Junyi Li, "Statistical Blockage Modeling and Robustness of Beamforming in Millimeter Wave Systems", ARXIV, pp.1-28, Janvier 2018. 
[8] Paolo Baracca, Andreas Weber, Thorsten Wild, Christophe Grangeat, "A Statistical Approach for RF Exposure Compliance Boundary Assessment in Massive MIMO Systems", ARXIV,pp.1-6, Janvier 2018.

[9] Guido Carlo Ferrante, Johan O stman, Giuseppe Durisi, and Kittipong Kittichokechai, "Pilot-Assisted Short-Packet Transmission over Multiantenna Fading Channels: A 5G Case Study", pp.1-6, Febraury 2018

[10] Alessio Zappone, Pin-Hsun Lin and Eduard Jorswieck," Optimal Energy-Efficient Design of Confidential Multiple-Antenna Systems", IEEE TRANSACTIONS ON INFORMATION FORENSICS AND SECURITY, Volume 13, issue 1, January 2018.

[11] Carlos A. Guti'errez, Jos'e T. Guti'errez-Mena, Jos'e M. Luna-Rivera, Daniel U. Campos-Delgado, Ramiro Vel'azquez, and Matthias P"atzold, "Geometry-Based Statistical Modeling of Non-WSSUS Mobile-toMobile Rayleigh Fading Channels ", IEEE TRANSACTIONS ON VEHICULAR TECHNOLOGY, Volume 67, Issue 1, January 2018.

[12] Hao Jiang, Zaichen Zhang, Jian Dang, Member and Liang Wu, "A Novel 3-D Massive MIMO Channel Model for Vehicle-to-Vehicle Communication Environments", IEEE TRANSACTIONS ON COMMUNICATIONS, Volume. 66, Issue 1, pp. 79 - 90, January 2018.

[13] Sha Hu, Fredrik Rusek, and Ove Edfors, "Beyond Massive-MIMO: The Potential of Positioning with Large Intelligent Surfaces", IEEE Transactions on Signal Processing, Volume PP Issue 99, pp. 1-14, January 2018.

[14] Callum T. Neil, Adrian Garcia-Rodriguez, , Peter J. Smith, Pawel A. Dmochowski, Christos Masouros and Mansoor Shafi, "On the Performance of Spatially Correlated Large Antenna Arrays for Millimeter-Wave Frequencies", IEEE TRANSACTIONS ON
ANTENNAS AND PROPAGATION, Volume 66, Issue 1, pp. 132- 148, January 2018.

[15] Guodong Li, Jinsong Wu, Zhixin Chen, Xiong Luo, Taolin Tang and Zhiqiang $\mathrm{Xu}$, "Performance analysis and evaluation for active antenna arrays under three-dimensional wireless channel model", IEEE Access, Volume PP, Issue 99, PP. 1-10, January 2018.

[16] Zhengyi Zhou, Ning Ge, Member, Zhaocheng Wang, Sheng Chen, "Hardware-Efficient Hybrid Precoding for Millimeter Wave Systems with Multi-Feed Reflectarrays", IEEE Access, Volume PP, Issue 99, PP. 1-11, January 2018.

[17] Qiang Hu, Meixiang Zhang, and Renzheng Gao, "Key Technologies in Massive MIMO ", 4th Annual International Conference on Wireless Communication and Sensor Network (WCSN 2017), Volume 17, Issue ITM Web conference, PP. 1-10 February 2018.

[18] Kui Xu, Zhexian Shen, Yurong Wang, Xiaochen Xia, "Beam-domain full-duplex transmission in massive MIMO system", Physical Communication, Volume 26, Issue 2018, pp.116-127, December 2017.

[19] Amr AkL, Ahmed Elnakib, Sherif Kishk, "Antenna array thinning for interference mitigation in multi-directional antenna subset modulation", Physical Communication, Volume 26, Issdue 2018, pp. 31-39, November 2017.

[20] Xuefeng Liu, Weixia Zou, "Block-sparse hybrid precoding and limited feedback for millimeter wave massive MIMO systems", Physical Communication, Volume 26, Issue 2018, pp. 81-86, December 2017.

[21] Mouloud Kamali, Adnen Cherif, "Adaptive Cylindrical Antenna Array For Massive MIMO in 5G", International Journal of Computer Science and Network Security, VOL.18 No.3, March 2018. 